\title{
Empyema Thoracis Caused by Drug-Resistant Staphylococcus aureus in an Adult Female: A Case Report
}

\author{
Roland Cheofor Ngu • Tee Ebogo-Ngwa • Vitalis Fambombi Feteh • \\ Christian Akem Dimala
}

Received: February 10, 2017 / Published online: March 20, 2017

(C) The Author(s) 2017. This article is an open access publication

\section{ABSTRACT}

Introduction: Empyema thoracis, which is the collection of pus in the pleural cavity, has been experiencing an increase in incidence rate in both adults and children. When present, it is most often a complication of some cases of pneumonia, but a proportion may result from iatrogenic causes or develop without pneumonia. It, therefore, constitutes a significant management challenge. In the case of bacteria

Enhanced content To view enhanced content for this article go to http://www.medengine.com/Redeem/ 44F7F0604BC2D501.

R. C. Ngu $(\varangle)$. V. F. Feteh

Mboppi Baptist Hospital, Douala, Cameroon

e-mail: rolinaro@gmail.com

R. C. Ngu · T. Ebogo-Ngwa · V. F. Feteh ·

C. A. Dimala

Medical Doctors Research Group (MDRG), Douala, Cameroon

R. C. Ngu · V. F. Feteh · C. A. Dimala

Health and Human Development (2HD) Research

Network, Douala, Cameroon

T. Ebogo-Ngwa

Faculty of Medicine and Biomedical Sciences,

University of Yaoundé, Yaoundé, Cameroon

C. A. Dimala

Faculty of Epidemiology and Population Health, London School of Hygiene and Tropical Medicine, London, UK resistant to conventional antibiotics, management of this potentially fatal complication may be difficult and is often associated with high morbidity and mortality (ranging from 6\% to $24 \%)$. We herein report a case of empyema thoracis caused by drug-resistant Staphylococcus aureus in an adult female.

Case Presentation: We report the case of a 28-year-old woman of black ethnicity who presented to the outpatient department with a 3 -week history of unproductive cough, sharp right-sided chest pain, shortness of breath, and easy fatigability. Physical examination revealed signs of right lung consolidation, and chest $\mathrm{X}$-ray showed a massive right pleural effusion with fluid analysis revealing of a purulent exudate and gram positive cocci in clusters, which was managed with cloxacillin. Culture had growth of multidrug-resistant $S$. aureus sensitive to clindamycin. A definitive diagnosis of empyema thoracis was made and treated with potent antibiotics and chest tube drainage with an initial positive outcome. There was a relapse a week later at the follow-up visit, necessitating further antibiotherapy and associations.

Conclusion: Empyema thoracis is a common complication, though not always, of longstanding pneumonia with $S$. aureus as one of the main causative agents. However, the resistance of this pathogen to conventional antibiotics probably due to longstanding self-medication makes management delicate/difficult, as in this case. We, therefore, recommend aggressive 
management, combining pus draining and intravenous anti-biotherapy, especially in this era of growing bacterial resistance to antibiotics.

Keywords: Drug resistant; Empyema thoracis; Staphylococcus aureus

\section{INTRODUCTION}

Empyema thoracis is a disease that despite centuries of study, still causes significant morbidity and mortality [1]. It is an infection of the pleural space (pus accumulation) that is most commonly a complication of pneumonia (parapneumonic). Complicated parapneumonic effusion or pleural empyema develops in $10-20 \%$ of outpatients with pneumonia [2]. Empyema thoracis has been traditionally categorized based on three clinical phases: exudative, fibrinopurulent, and organizing [3-5]. The initial diagnosis is made after a thorough history and physical exam to delineate the start of symptoms and prior antibiotic treatments. The standard chest X-ray is usually the first radiographic study obtained [2]. The management of empyema thoracis usually depends on the clinical phase. Multiple therapeutic options are available for the management of empyema: antibiotherapy, thoracentesis, closed (tube) thoracostomy, image-guided catheter drainage, and decortication; however, the optimal therapeutic management has not been elucidated $[3,4]$. Decortication is more frequently necessary for anaerobic, tuberculous, staphylococcal, and pneumococcal infections [4]. When dealing with staphylococcal empyema, treatment should be directed into three main channels: (1) control of the infecting organism; (2) obliteration of the empyema space; and (3) general supportive measures. With the emergence of an increasing number of strains resistant to the commonly used antibiotics, it seemed worthwhile to review our recent cases of empyema with particular emphasis on the bacteriological aspects [6]. Data are limited on antibiotic resistance of some of the common causative organisms implicated in the formation of empyema thoracis in developing settings. Here we report the case of empyema thoracis caused by drug-resistant Staphylococcus aureus in an adult female.

\section{CASE PRESENTATION}

This is the case of a 28-year-old female farmer of black ethnicity who presented to the outpatient department with a 3-week history of unproductive cough, sharp right-sided chest pain, and shortness of breath, all exacerbated by mild exertion. Symptoms were associated with low grade fever, night sweats, weight loss, and anorexia. She was a non-smoker, seronegative for human immunodeficiency virus (HIV), no history of diabetes or use of corticosteroids or other medications with immunosuppressive potentials, and also denied any tuberculosis (TB) exposure. Prior to consultation she had taken as self-medication a suboptimal dose oral amoxicillin and cotrimoxazole.

On examination, she was conscious and had labored breathing with a respiratory rate of 32 breaths per minute, temperature $37.5^{\circ} \mathrm{C}$, heart rate 100 beats per minute, a blood pressure of $100 / 60 \mathrm{mmHg}$, and oxygen saturation of $93 \%$. She had pink conjunctivae, anicteric sclerae, and no central or peripheral cyanosis. Her chest exam revealed a decreased tactile vocal fremitus and dull percussion note on the right lung field, decreased breath sounds on the left lung and lower third of the right lung field with no crackles nor muffled heart sounds. The abdominal examination was normal. Her full blood count revealed a white blood cell count of $5.9 \times 10^{3} / \mu \mathrm{L}$ ), lymphocytes $3.2 \times 10^{3} / \mu \mathrm{L}$, granulocytes $2.4 \times 10^{3} / \mu \mathrm{L}$, mean corpuscular volume $(\mathrm{MCV}) \quad 92 \mathrm{fL}$, mean corpuscular hemoglobin concentration (MCHC) $32 \mathrm{~g} / \mathrm{dL}$, hematocrit of $28.5 \%$, hemoglobin (Hb) of $9.5 \mathrm{~g} /$ dL (normochromic normocytic anemia), platelet count of $208 \times 10^{3} / \mu \mathrm{L}$. Zeihl-Neilsen stain of sputum revealed no acid fast bacilli (AFB) on two samples. Chest X-ray showed extensive lower lung field concave opacity suggestive of a massive right pleural effusion (Fig. 1).

She was diagnosed of empyema thoracis and started on empiric antibiotherapy with intravenous (IV) cloxacillin, metronidazole, diclofenac tablets, IV normal saline, and a right 


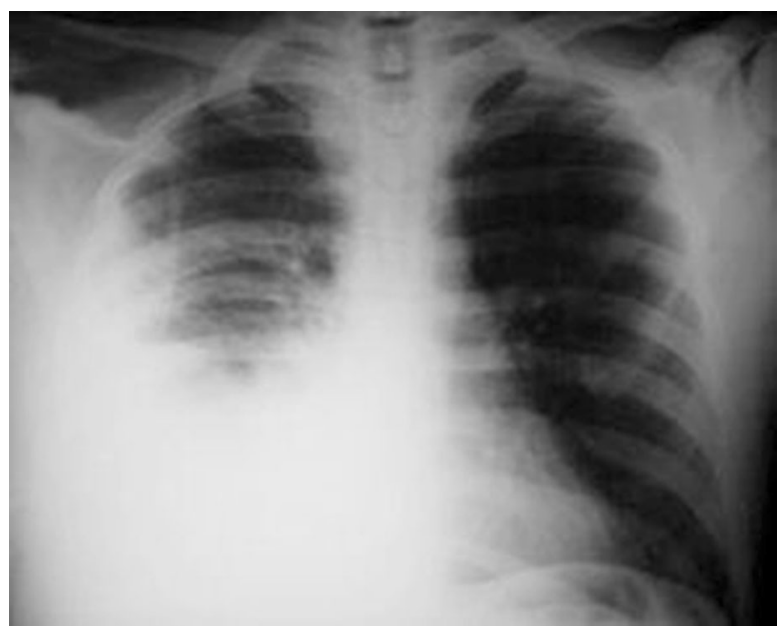

Fig. 1 Chest X-ray showing massive right pleural effusion

thoracotomy was done to insert a chest tube connected to an underwater seal drain with an initial drainage of $2.5 \mathrm{~L}$ of purulent fluid, which was sent for analysis and culture. Pleural fluid analysis showed a purulent fluid, positive for proteins $(300 \mathrm{mg} / \mathrm{dL})$, glucose of $25 \mathrm{mg} / \mathrm{dL}$, differential neutrophil and lymphocyte percentages of $65 \%$ and $35 \%$, respectively, massive white blood cells (WBC) count per high power field $\left(2020 / \mathrm{mm}^{3}\right)$, gram stain revealed the presence of gram positive cocci in clusters. Lactate dehydrogenase (LDH) and $\mathrm{pH}$ of the pleural fluid weren't analyzed by the laboratory. The chest tube was left in situ with lavage of pleural cavity with normal saline $0.9 \%$ on alternate days and was well safeguarded to avoid an iatrogenic pneumothorax or subcutaneous emphysema and fluid in the drainage can was monitor 24 hourly and discarded (similar to pleural irrigation trial by Hooper et al. though not exactly the same) [7]. On day 3 of admission, pus culture showed significant growth of multidrug-resistant $S$. aureus resistant to cloxacillin, but sensitive to clindamycin, vancomycin, and ciprofloxacin leading to a switch of the IV cloxacillin to oral clindamycin. The chest tube was removed on the seventh day of admission when drainage was no longer significant, and a control X-ray showed marked reduction of pleural effusion (Fig. 2). She was discharged on the eighth day after chest tube removal on oral

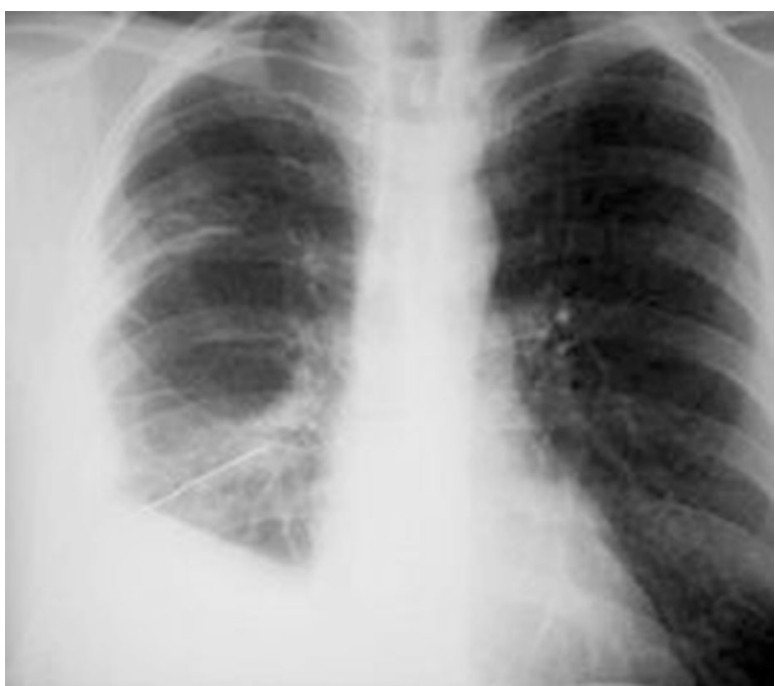

Fig. 2 Control chest X-ray showing reduction of pleural effusion

amoxicillin plus clavulanic acid, clindamycin, metronidazole, and diclofenac.

A week later she came for follow-up still complaining of right-sided chest pain and shortness of breath, and another control X-ray showed mild to moderate pleural effusion, which again showed gram positive cocci in clusters on analysis. She was given $1.2 \mathrm{~g}$ of cefuroxime given IV for 5 days, asked to continue with her ongoing medication, and given another appointment in a week's time, but she didn't show up.

Informed consent was obtained from the patient for publication of this case report and any accompanying images. All procedures followed were in accordance with the ethical standards of the responsible committee on human experimentation (institutional and national) and with the Helsinki Declaration of 1964, as revised in 2013.

\section{DISCUSSION}

Empyema, which is pus accumulation in the pleural cavity has a three-stage natural history: the exudative, fibrinopurulent (stage of empyema thoracis), and organizing phases [8]. Empyema may originate from parapneumonic pleural effusion (stage-I usually present for 1 to 
3 days) when fluid may still be sterile with low leukocyte count $(<50 \%)$, low LDH levels, with a $\mathrm{pH}$ in normal physiological range, and usually normal glucose levels. If not treated early with appropriate antibiotics it may progress to stage-II (fibropurulent, i.e., last 4-14 days) with high leukocytes, high $\mathrm{LDH}$, low $\mathrm{pH}(<7)$, and low glucose, which the most likely stage of this patient, but couldn't be confirmed 100\% due to lack of LDH and $\mathrm{pH}$ in the pleural fluid analysis result. If still not treated with antibiotics or fibrinolytics and intercostal drainage (closed tube thoracostomy), as in the management of this case, it may progress to organizational stage; stage-III usually occurs beyond 14 days, with extensive fibrositic activity and fibrin deposition, with loculation and septation. Pus formation under pressure may track its way to adjacent structures or to the chest wall [9].

Empyemas develop primarily because of delayed presentation by the patient with advanced pneumonia and progressive pleural infection as was the case in this patient and less often, from inappropriate clinical management. Early antibiotic treatment prevents progression of pneumonia to parapneumonic pleural effusion (PPE) and thus to empyema [10]. In our patient, delayed presentation was due to self-medication of an inappropriate dose of over-the-counter antibiotherapy, which is readily available in Cameroon and is known to be one of the main drivers of the antibiotic resistance pandemic [11].

In most series of patients with community acquired empyema, aerobic bacteria predominate. These include Streptococcus pneumoniae and $S$. aureus (commonest cause of empyema, which was the micro-organism identified in this case) often easily treatable with adequate antibiotherapy. Aerobic organisms also include gram negative bacteria such as Escherichia coli, Haemophilus influenza, and Klebsiella pneumoniae [12]. Mixed aerobic and anaerobic bacteria are commonly isolated from empyema. The commonest anaerobes are Bacteroides fragilis [13].

The symptoms of pneumonia involving a PPE or empyema (i.e., fever, malaise, cough, dyspnea, and pleuritic chest pain) are similar to those of pneumonia without a PPE. Our patient probably had pneumonia due to inappropriate treatment that progressed to pneumonia with PPE. Elderly patients may be relatively asymptomatic, presenting only with fatigue or altered mental status, without pulmonary symptoms, making suspicion and diagnosis relatively more difficult in such situations. However, that was not the situation in this patient since she was 28 years old. Other factors, such as age, peak temperature, leukocyte count, or number of lobes involved, cannot predict the presence of a PPE or differentiate between persons with and persons without a PPE. The clinical examination of empyema patients often reveals stony dull percussion note, diminished or absent air entry in the lung, diminished tactile vocal fremitus, and vocal resonance of the lung, as was the case in this patient. Empyema patients usually represent a neglected pleural infection. Patients who are hospitalized with a PPE and who receive appropriate antibiotic treatment rarely $(<2 \%)$ develop empyema [14].

Laboratory Investigations used in the diagnosis of empyema thoracis include the following. Chest X-rays and/or computerize tomography (CT) scans (very scarce in Cameroon and wasn't done in this patient) that will show whether or not there's fluid in the pleural space, as in this case. An ultrasound of the chest is used to see the amount of fluid and its exact location. Blood tests are used to check for white blood cell count and C-reactive protein and to identify the particular bacteria causing the infection. White cell count is usually elevated when you have an infection. But this patient had a normal white cell count, which could suggest a decreased immune reaction to the longstanding infection. On the other hand this patient had a normochromic normocytic anemia, which supports the possibility of chronic inflammation in a longstanding infection. There was no reported history of menstruation or blood loss from other sources at the time of presentation to us. Other investigations such as sputum analysis by Ziehl-Neelson stain for AFB and gram stain or blood culture can also be done to identify causative organisms. During a thoracentesis, a needle is inserted through the back of the rib cage into the pleural space to take a sample of fluid for analysis and culture. Fluid analysis under a microscope looks for 
bacteria, protein, and other cells, as seen in this patient [15]. We ensured that the thoracocentesis to collect pleural fluid was done before initiating antibiotics, which if done otherwise could alter our results [16].

Treatment is aimed at removing the pus, draining the fluid from the pleural space (closed tube thoracostomy and treating the infection). Antibiotics are used to treat the underlying infection. The specific type of antibiotic depends on what type of bacteria is causing the infection. A culture of the collected fluid is ideally done to confirm the sensitivity of the causative pathogens to specific antibiotics. Given the acute presentation in our patient, we started the patient immediately on antibiotics while waiting for the culture results. The $S$. aureus isolated in this case by culture was resistant to multiple antibiotics. Drug resistance is a growing public health problem with potential ramification on the cost of health care, especially in low resource settings such as Cameroon where infectious diseases are still a significant cause of morbidity and mortality. Resistance in this case could be explained by the fact that the patient had taken several over-the-counter antibiotics before coming to the hospital. The culture results obtained on day 3 of admission identified $S$. aureus resistant to cloxacillin and an array of antibiotics and sensitive to clindamycin. We then decided to switch the cloxacillin to clindamycin oral, but 1 week after she was discharged, symptoms relapsed despite treatment with clindamycin.

The next step is to drain the pus, and the specific method of pleural fluid drainage depends on the stage of the empyema. In simple empyema, a needle can be inserted into the pleural space to drain the fluid. This is called percutaneous thoracentesis [15]. In the later stages (complex empyema), a drainage tube must be used to drain the pus (closed tube thoracotomy). This procedure is usually performed under anesthesia in an operating room. We, therefore, performed a closed-tube thoracotomy in our case, given the fact that the effusion was massive and the draining was to be done progressively over hours to days.
More modern techniques such as video-assisted thoracic surgery (VATS) permits us to remove the affected tissue around the lung and insert a drainage tube or use medication to remove the fluid. However, given the limited array of facilities/equipment in our setting we could not perform VATS.

\section{CONCLUSION}

Empyema thoracis should be suspected in patients with longstanding pneumonia or pneumonia unresponsive to appropriate antibiotherapy. A chest radiograph (X-ray and/ or CT scan) should be done to make the diagnosis. Complementary investigations such as ultrasound scan could be done to quantify the amount of fluid in the intrapleural space, as well as a pleural fluid analysis and culture is essential to identify causative organisms in order to plan appropriate treatment though there may be multi-drug resistance by some organisms like in this case. Thus, prompt recognition and timely management of pneumonia will prevent development of parapneumonic pleural effusion and thus empyema thoracis.

\section{ACKNOWLEDGEMENTS}

We will like to thank the patient and her family for their co-operation towards making this publication and also Babo Aline for her inspiring support towards writing this paper. No funding or sponsorship was received for this study or publication of this article. All named authors meet the International Committee of Medical Journal Editors (ICMJE) criteria for authorship for this manuscript, take responsibility for the integrity of the work as a whole, and have given final approval to the version to be published.

Disclosures. Roland Cheofor Ngu, Tee Ebogo-Ngwa, Vitalis Fambombi Feteh, and Christian Akem Dimala declare that they have no competing interests. 
Compliance with Ethics Guidelines. Informed consent was obtained from the patient for publication of this case report and any accompanying images. All procedures followed were in accordance with the ethical standards of the responsible committee on human experimentation (institutional and national) and with the Helsinki Declaration of 1964, as revised in 2013.

Data Availability. All available data are included within the manuscript.

Open Access. This article is distributed under the terms of the Creative Commons Attribution-NonCommercial 4.0 International License (http://creativecommons.org/licenses/ by-nc/4.0/), which permits any noncommercial use, distribution, and reproduction in any medium, provided you give appropriate credit to the original author(s) and the source, provide a link to the Creative Commons license, and indicate if changes were made.

\section{REFERENCES}

1. Acharya PR, Shah KV. Empyema thoracis: a clinical study. Ann Thorac Med. 2007;2:14-7.

2. Wait MA, Beckles DL, Paul M, Hotze M, DiMaio MJ. Thoracoscopic management of empyema thoracis. J Minim Access Surg. 2007;3:141-8.

3. de Souza A, Offner PJ, Moore EE, Biffl WL, Haenel JB, Franciose RJ, et al. Optimal management of complicated empyema. Am J Surg. 2000;180:507-11.

4. Mandal AK, Thadepalli H, Mandal AK, Chettipally U. Outcome of primary empyema thoracis: therapeutic and microbiologic aspects. Ann Thorac Surg. 1998;66:1782-6.

5. Lackner RP, Hughes R, Anderson LA, Sammut PH, Thompson AB. Video-assisted evacuation of empyema is the preferred procedure for management of pleural space infections. Am J Surg. 2000;179:27-30.

6. Jewett TC, Carberry DM, Neter E. Staphylococcal empyema in children. Ann Surg. 1961;153:447-52.

7. Hooper CE, Edey AJ, Wallis A, Clive AO, Morley A, White $P$, et al. Pleural irrigation trial (PIT): a randomised controlled trial of pleural irrigation with normal saline versus standard care in patients with pleural infection. Eur Respir J. 2015;46:456-63.

8. Ahmed AEH, Yacoub TE. Empyema Thoracis. Clin Med Insights Circ Respir Pulm Med. 2010;4:1-8.

9. Chaudhry LA, Ba AA, Zamzami M, Robert AA. Contemporary empyema thoracic necessitans in an adult male caused by Staphylococcus aureus: decortication is superior to traditional under water seal intercostal tube in chronic empyema. Pan Afr Med J. 2015 [cited 2016 May 12]; 20. http://www. panafrican-med-journal.com/content/article/20/ 115/full/.

10. Sahn SA. Diagnosis and management of parapneumonic effusions and empyema. Clin Infect Dis. 2007;45:1480-6.

11. World Health Organization, editor. Antimicrobial resistance: global report on surveillance. Geneva, Switzerland: World Health Organization; 2014.

12. Brook I, Frazier EH. Aerobic and anaerobic microbiology of empyema. A retrospective review in two military hospitals. Chest. 1993;103:1502-7.

13. Civen R, Jousimies-Somer H, Marina M, Borenstein L, Shah H, Finegold SM. A retrospective review of cases of anaerobic empyema and update of bacteriology. Clin Infect Dis Off Publ Infect Dis Soc Am. 1995;20(Suppl 2):S224-9.

14. Sahn SA. Diagnosis and management of parapneumonic effusions and empyema. Clin Infect Dis. 2007;45:1480-6.

15. Strachan RE, Cornelius A, Gilbert GL, Gulliver T, Martin A, McDonald T, et al. Bacterial causes of empyema in children, Australia, 2007-2009. Emerg Infect Dis. 2011;17:1839-45.

16. Balachandran A, Shivbalan S, Thangavelu S, Vijayasekaran D, Subramnayam L. Empyema thoracis. Indian J Pediatr. 2003;70:803-6. 